\title{
Wavelet-Based Estimation of Long-Range Dependence in MPEG Video Traces
}

\author{
Nikola Cackov and Želimir Lučić \\ School of Engineering Science \\ Simon Fraser University \\ Vancouver, Canada \\ \{ncackov, zlucic\}@cs.sfu.ca
}

\author{
Momčilo Bogdanov \\ Faculty of Electrical Engineering \\ Saints Cyril and Methodius University \\ Skopje, Macedonia \\ bogdanov@etf.ukim.edu.mk
}

\author{
Ljiljana Trajković \\ School of Engineering Science \\ Simon Fraser University \\ Vancouver, Canada \\ ljilja@cs.sfu.ca
}

\begin{abstract}
Correct and efficient estimation of the Hurst parameter of long-range dependent (LRD) video traces is important in traffic analysis. The low computational cost and the wavelets' scale invariance make the wavelet transform suitable for analysis of LRD processes. In this paper, we apply wavelet-based estimation of the Hurst parameter to MPEG-1 and MPEG-4 encoded video sequences. Frequency-domain estimators (periodogram and wavelet-based) produce different Hurst parameters compared to time-domain estimators ( $R / S$ and variance-time plot). Waveletbased estimators often produce Hurst parameters that are close to or greater than one. Our analysis indicates that possible causes for the unreliable performance of the wavelet-based estimators are the non-stationarity of the scaling exponent and the existence of both short-range and long-range dependent components in the video traces.
\end{abstract}

\section{INTRODUCTION}

Long-range dependence (LRD) is often found in network traffic aggregated from multiple sources [1]. Several studies of the statistical properties of video sequences encoded using MPEG-1, MPEG-4, H.263, and proprietary algorithms [2]-[4] show that LRD is also present in variable bit-rate video traffic sources, regardless of the employed encoding algorithm.

The Hurst parameter $(H)$ is an important parameter that characterizes the level of LRD. Its correct and efficient estimation is important in statistical analysis. Known estimators for $H$ are based on either time-domain characteristics of longrange dependent processes (R/S plot and variance-time plot) or frequency spectrum characteristics (Whittle, periodogram, and wavelet-based estimators [5], [6]).

Application of the wavelet-based estimator of $H$ to network traffic has been already reported [6]. The estimator has also been used for video traces [4] and it often yielded nonphysical values of $H>1$. In this paper, we revisit the issue of the unreliable performance of the estimator when applied to MPEG-1 and MPEG-4 video traffic traces. The graphical outputs of the wavelet estimator indicate presence of LRD over the coarser time scales. However, numerical estimates of $H$ often lead to $H>1$. In this paper, we compare these results with estimates obtained from $\mathrm{R} / \mathrm{S}$ and periodogram estimators. We investigate the possible causes of the unreliability of the wavelet-based estimator by testing the Gaussianity

This research was supported by the NSERC Grant 216844-03 and Canada Foundation for Innovation New Opportunities program. of the wavelet coefficients and the time constancy of the scaling exponent $\alpha$. Furthermore, we address the unreliable performance of the wavelet-based Hurst parameter estimator when applied to traces that possess both strong LRD and shortrange dependent (SRD) components [7].

In Section II, we define long-range dependence and describe the fundamental properties of LRD processes. Video traffic traces and analysis tools are described in Section III. We present the analysis results in Section IV. Possible causes for the unreliable estimates of the Hurst parameter are investigated in Section V. We summarize our findings in Section VI.

\section{LONG-RANGE DEPENDENCE}

Let $X(n), n=0,1, \ldots$, be a wide-sense stationary stochastic process with an autocorrelation function $r(k)$ with time lag $k$. The process $X(n)$ is called long-range dependent if the sum of $r(k)$ over all $k$ 's is infinite [8]. For large lags $k, r(k)$ is modeled as a hyperbolically (power-law) decaying function:

$$
r(k)=c_{r} k^{-(2-2 H)}, \quad k \rightarrow \infty,
$$

where $c_{r}$ is a positive constant and $H(0.5 \leq H<1)$ is the Hurst parameter. The power spectral density (PSD) $f(\nu)$ of $X(n)$ satisfies

$$
f(\nu)=c_{f}|\nu|^{-\alpha},|\nu| \rightarrow 0,
$$

where $c_{f}$ is a positive constant and $\alpha$ is the scaling exponent [5]. For LRD processes, $0<\alpha<1$. The relationship between $H$ and $\alpha$ is linear:

$$
H=0.5(1+\alpha) \text {. }
$$

The Hurst parameter measures the degree of LRD of a process. For SRD processes, $H=0.5$. Values of $H \approx 1$ indicate a process with strong LRD. For example, bursty network traffic has a large $H$ [1].

\section{Video TRAFFIC TRACES AND ANALYsis TOOLS}

We analyze publicly available video traces [9], [10] that consist of sizes of video frames in bits. The duration of each frame is $40 \mathrm{~ms}$ ( 25 frames per second). The length of the traces varies between $15 \mathrm{~min}(22,498$ frames) and $60 \mathrm{~min}(89,998$ frames). A list of properties of each trace (video sequence, encoding algorithm, number of frames, and duration) is given in Table I. 
TABLE I

Properties of the Video Traces and Estimates of the Hurst Parameter.

\begin{tabular}{|c|c|c|c|c|c|c|c|c|c|}
\hline \multirow{3}{*}{ Trace } & \multirow{3}{*}{ Encoding } & \multirow{3}{*}{$\begin{array}{c}\text { Length } \\
\text { (frames) }\end{array}$} & \multirow{3}{*}{$\begin{array}{c}\text { Duration } \\
\text { (min) }\end{array}$} & \multicolumn{6}{|c|}{ Estimates of $\boldsymbol{H}$} \\
\hline & & & & \multicolumn{2}{|c|}{ Monofractal } & \multicolumn{2}{|c|}{ Multifractal } & \multirow{2}{*}{$\begin{array}{c}\text { Perio- } \\
\text { dogram }\end{array}$} & \multirow{2}{*}{$R / S$} \\
\hline & & & & range & value & range & value & & \\
\hline MTV & MPEG-1 & 40,000 & 26.67 & $4-12$ & 0.959 & $3-12$ & 0.937 & 0.992 & 0.89 \\
\hline Jurassic park & MPEG-1 & 40,000 & 26.67 & $5-12$ & 1.096 & $4-12$ & 1.012 & 1.191 & 0.88 \\
\hline Simpsons & MPEG-1 & 40,000 & 26.67 & $5-12$ & 0.926 & $4-12$ & 0.906 & 0.988 & 0.89 \\
\hline Mr. Bean & MPEG-1 & 40,000 & 26.67 & $5-12$ & 1.214 & $5-12$ & 1.258 & 1.295 & 0.85 \\
\hline Silence of the lambs & MPEG-1 & 40,000 & 26.67 & $5-12$ & 1.130 & $5-12$ & 1.152 & 1.171 & 0.89 \\
\hline Talk show & MPEG-1 & 40,000 & 26.67 & $5-12$ & 1.084 & $5-12$ & 1.132 & 1.174 & 0.89 \\
\hline ARD news & MPEG-4 & 22,498 & 15.00 & $5-11$ & 1.382 & $4-11$ & 1.225 & 1.310 & 0.967 \\
\hline Diehard III & MPEG-4 & 89,998 & 60.00 & $4-13$ & 1.190 & $4-13$ & 1.208 & 1.233 & 0.969 \\
\hline Formula 1 & MPEG-4 & 44,998 & 30.00 & $4-12$ & 1.189 & $4-12$ & 1.169 & 1.216 & 0.867 \\
\hline Futurama & MPEG-4 & 30,334 & 20.22 & $4-12$ & 0.943 & $4-12$ & 0.909 & 1.064 & 0.877 \\
\hline From dusk till dawn & MPEG-4 & 89,998 & 60.00 & $4-13$ & 1.139 & $4-13$ & 1.138 & 1.186 & 0.909 \\
\hline First contact & MPEG-4 & 89,998 & 60.00 & $4-13$ & 1.194 & $4-13$ & 1.213 & 1.268 & 0.931 \\
\hline Mr. Bean & MPEG-4 & 89,057 & 59.37 & $4-13$ & 1.083 & $4-13$ & 1.109 & 1.151 & 0.933 \\
\hline Jurassic park & MPEG-4 & 89,998 & 60.00 & $4-13$ & 1.222 & $4-13$ & 1.247 & 1.293 & 0.973 \\
\hline VIVA video clips & MPEG-4 & 89,998 & 60.00 & $2-13$ & 1.000 & $2-13$ & 1.120 & 1.119 & 0.961 \\
\hline N3 talk & MPEG-4 & 89,998 & 60.00 & $4-13$ & 1.079 & $4-13$ & 1.131 & 1.188 & 0.882 \\
\hline Silence of the lambs & MPEG-4 & 89,998 & 60.00 & $4-13$ & 1.277 & $4-13$ & 1.260 & 1.337 & 1.007 \\
\hline Simpsons & MPEG-4 & 30,334 & 20.22 & $4-12$ & 0.964 & $4-12$ & 0.941 & 1.061 & 0.889 \\
\hline Star Wars IV & MPEG-4 & 89,998 & 60.00 & $4-13$ & 1.013 & $4-13$ & 1.051 & 1.138 & 0.903 \\
\hline
\end{tabular}

\section{A. Discrete Wavelet Transform}

The discrete wavelet transform (DWT) represents a signal $X(t)$ as a weighted sum of basis functions called wavelets [5]:

$$
X(t)=\sum_{j=0}^{\infty} \sum_{k=-\infty}^{\infty} d(j, k) \psi_{j, k}(t),
$$

where $d(j, k)$ is the wavelet coefficient at octave $j$ and time $k$ and

$$
\psi_{j, k}(t)=2^{-j / 2} \psi\left(2^{-j} t-k\right)
$$

is the wavelet obtained from an adequately chosen mother wavelet $\psi$. The wavelet $\psi_{j, k}$ is a scaled (by a factor of $2^{-j}$ ) and shifted (by $k$ time units) version of the mother wavelet.

The DWT captures a signal at various time scales (levels of aggregation). Due to the scale invariance of the basis functions, it is suitable for analyzing properties that are present across a range of time scales, such as LRD. The DWT may be implemented by a filter-bank-based pyramidal algorithm. Its low computational cost makes the DWT a popular tool for signal analysis [11].

\section{B. Wavelet-based Hurst Parameter Estimator}

The wavelet-based Hurst parameter estimator is based on the shape of the PSD function (2) of the LRD signal $X(n)$. It has been shown [5], [6] that when the PSD has a power-law behavior, the relationship between the variance of the wavelet coefficients on a given octave and the octave $j$ is

$$
\mathrm{E}\left\{d(j, k)^{2}\right\}=2^{j \alpha} c_{f} C,
$$

where the average is calculated for various $k$ and $C$ is a constant that depends on the choice of the mother wavelet.
When a suitable mother wavelet is chosen [5], calculation of $\mathrm{E}\left\{d(j, k)^{2}\right\}$ becomes a simple time average or sample mean for all $k$ 's:

$$
\mathrm{E}\left\{d(j, k)^{2}\right\}=\frac{1}{n_{j}} \sum_{k=1}^{n_{j}} d(j, k)^{2},
$$

where $n_{j}$ is the number of wavelet coefficients available at octave $j$. Linear relationship with a slope $\alpha(0<\alpha<1)$ between $\log _{2} \mathrm{E}\left\{d(j, k)^{2}\right\}$ and $j$ for a range of octaves, including the coarsest, indicates presence of LRD. Therefore, $\alpha$ is obtained by performing linear regression of $\log _{2} \mathrm{E}\left\{d(j, k)^{2}\right\}$ over $j$ in a range of octaves. $H$ is calculated by using (3). We employ publicly available MATLAB code [12] to compute the estimates of $\log _{2} \mathrm{E}\left\{d(j, k)^{2}\right\}$, variances of the estimates, and to perform weighted linear regression. The weights are inversely proportional to the variances of the estimates of $\log _{2} \mathrm{E}\left\{d(j, k)^{2}\right\}$. This estimator is called monofractal wavelet estimator.

An extension to the basic monofractal wavelet estimator is the multifractal estimator [13]. In addition to the second moments (variances) of the wavelet coefficients, it also takes into account moments of higher order:

$$
S_{q}(j)=\frac{1}{n_{j}} \sum_{k=1}^{n_{j}} d(j, k)^{q} .
$$

It estimates the slope $\alpha_{q}$ by performing linear regression of $\log _{2} S_{q}(j)$ for a range of $j$ 's. $H$ is calculated using an expression analogous to (3), by taking into account the order of the moment:

$$
H=0.5+\alpha_{q} / q .
$$


Both monofractal and multifractal estimators are used to produce diagrams of $\log _{2} \mathrm{E}\left\{d(j, k)^{2}\right\}$ or $\log _{2} S_{q}(j)$ versus $j$, with the corresponding confidence intervals. They are called logscale diagrams. An example is shown in Fig. 1. The solid line connects the estimates of $\log _{2} \mathrm{E}\left\{d(j, k)^{2}\right\}$ and the dashed line is the slope of the linear regression performed over the range of octaves [4-13]. The vertical lines represent the confidence intervals of the estimates of $\log _{2} \mathrm{E}\left\{d(j, k)^{2}\right\}$.

\section{Test for Time Constancy of the Scaling Exponent $\alpha$}

LRD processes are wide-sense stationary. However, they exhibit high burstiness and appear non-stationary [14]. We test the time constancy of the scaling exponent $\alpha$ to determine whether a process is wide-sense stationary with LRD or inherently non-stationary.

The definition and the statistical properties of the test for the constancy of $\alpha$ are given in [14]. The trace is divided into $m$ sub-traces and $\alpha$ is estimated for each sub-trace. The estimates are then compared and a decision is made whether or not $\alpha$ may be considered constant over the duration of the entire trace. The test, implemented in MATLAB, is available online [12].

\section{Hurst Parameter Estimation}

We estimate the Hurst parameter of nineteen video traffic traces using both monofractal and multifractal wavelet-based estimators. We employ Daubechies' wavelets of genus 3 (three vanishing moments). The results are summarized in Table I. For each estimate of $H$, we report the range of octaves where the linear regression is performed. These ranges are chosen by visual inspection of the logscale diagrams and identification of the linear region. For the multifractal estimator, we choose the order of the moments $q=3$. Also shown in Table I are estimates of $H$ obtained from the periodogram-based estimator [15] and R/S plots [3], [10].

Logscale diagrams of the analyzed traces have similar shapes. A typical example is shown in Fig. 1. Logscale diagrams exhibit a linear relationship between $\log _{2} \mathrm{E}\left\{d(j, k)^{2}\right\}$ $\left(\log _{2} S_{q}(j)\right)$ and $j$ for the largest values of $j$ (the coarsest octaves or time scales). The linear region typically begins at $j=4$ or 5 . The lack of linearity for the finer octaves may be attributed to artifacts of MPEG compression algorithms or to a transition between short-term and long-term scaling behavior [4].

Both monofractal and multifractal estimators of $H$ produce similar results, as indicated by Table I. They are in good agreement with periodogram-based estimates. On the contrary, a majority of wavelet and periodogram-based estimates are greater than one and differ from R/S estimates. The linearity of the logscale diagrams for the coarsest octaves and the good match between wavelet-based and periodogram-based estimates indicate that PSDs of the traces exhibit power-law behavior close to the origin, with exponents $\alpha>1$. This contradicts the LRD assumption because for LRD processes $\alpha<1$.

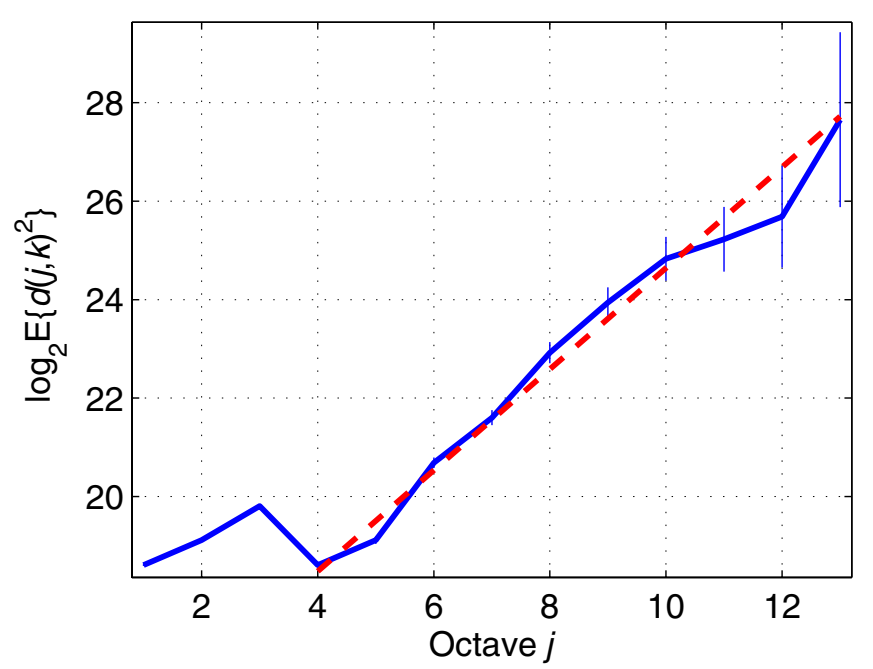

Fig. 1. Logscale diagram of the MPEG-4 encoded "Star Wars IV" video sequence.

\section{InVESTIGATING THE SOURCES OF UNRELIABILITy OF THE ESTIMATES}

In order to investigate the possible sources of the unreliability of the wavelet estimators, we test the Gaussianity of the traces and the time constancy of the scaling exponent $\alpha$. We also address the performance of the monofractal waveletbased estimator in the presence of strong SRD and LRD components [7].

\section{A. Testing the Gaussianity of the Wavelet Coefficients}

One of the idealizations assumed by the wavelet estimator is that the analyzed process and its wavelet coefficients on various octaves are Gaussian [5]. Therefore, we use $q-q$ plots [16] to examine how close the traces and their wavelet coefficients are to a Gaussian distribution. A sample set of q-q plots is shown in Fig. 2. The dashed line is the reference line with a slope of one. The vertical lines mark the $10 \%$ and $90 \%$ quantiles. The wavelet coefficients are approximately Gaussian in the range of octaves where $H$ is estimated.

\section{B. Testing the Time Constancy of $\alpha$}

We examine the time constancy of $\alpha$ for each trace. We perform a set of tests by varying the number of sub-traces $(m)$ between 3 and 15. The lower bound of the range of octaves where $\alpha$ is estimated is set to the value given in Table I. It varies between 2 and 5 . The upper bound depends on $m$. For larger $m$, the sub-traces are shorter and there are fewer available octaves. In our experiments, the upper octave varies between 8 and 12. A sample graphical output of the test for $m=12$ is shown in Fig. 3. The graph shows the overall value of $\alpha$ (solid horizontal line), the average of the 12 estimates of $\alpha$ (dashed horizontal line), and the confidence intervals of the estimates. In this example, the test shows that the probability of $\alpha$ being constant is $0 \%$.

Our findings indicate that twelve traces fail the test for all values of $m$, while others pass the test for certain values of $m$ 

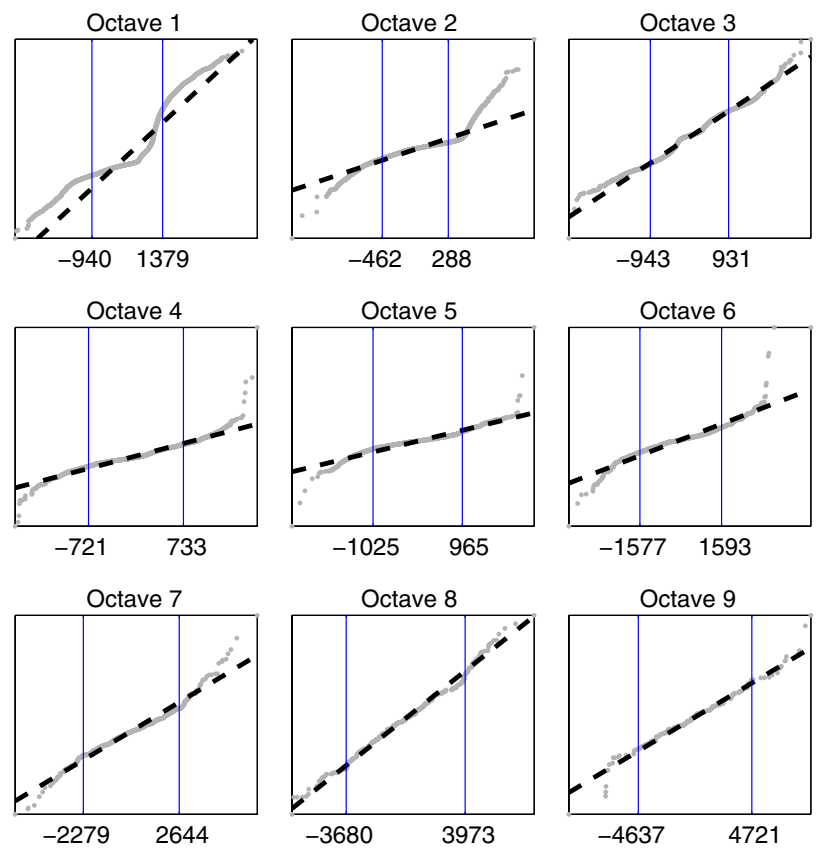

Fig. 2. Q-Q plots of the wavelet coefficients for octaves 1-9 of the "Star Wars IV" video sequence.

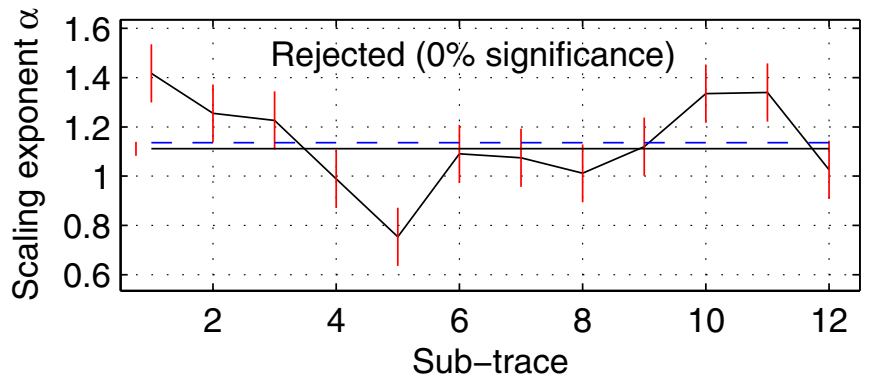

Fig. 3. Test for time constancy of $\alpha$ for the MPEG-4 encoded "Star Wars IV" video sequence.

and fail for others. MPEG-1 encoded "Simpsons" and MPEG4 encoded "ARD news" video sequences pass the test for over $50 \%$ of $\mathrm{m}$ 's. The remaining seven video traces pass the test for less than $50 \%$ of $m$ 's This indicates that $\alpha$ is not constant and varies with time. Therefore, estimating $\alpha$ and $H$ for the entire trace may not produce reliable results.

\section{Possible Causes of the Unreliable Estimates}

Presented results show that the wavelet-based estimators produce values of $H>1$. The traces often fail the test for time constancy of $\alpha$. The linearity of the logscale diagrams may be attributed to the averaging of the non-stationarities that manifest as variability of the scaling exponent $\alpha$ across a trace [14].

Estimates of $H$ obtained from R/S plots are often greater than 0.9 , which indicates strong LRD component. We conjecture that video traces also possess a strong SRD component. Video sequences consist of various scenes. Video frames representing a single scene are similar due to the identical or similar background and objects present in the scene. This implies similar sizes of the adjacent frames, which indicates a strong positive correlation for small lags. It has been shown that the monofractal wavelet-based estimator produces unreliable results when applied to processes with a strong SRD component and a strong LRD component [7].

\section{CONCLUSION}

We applied wavelet-based estimators of the Hurst parameter to MPEG-1 and MPEG-4 encoded video traces. Both monofractal and multifractal estimators produced similar results. The estimates of the Hurst parameter were often greater than one. We investigated the possible causes of the unreliable results by examining the distribution of the wavelet coefficients and the time constancy of the scaling exponent $\alpha$. Our findings indicated that the unreliable performance of the estimators may be attributed to the variability of the exponent $\alpha$ across the trace and to the presence of strong SRD and LRD components.

\section{REFERENCES}

[1] W. Leland, M. Taqqu, W. Willinger, and D. Wilson, "On the selfsimilar nature of Ethernet traffic (extended version)," IEEE/ACM Trans. Networking, vol. 2, pp. 1-15, Feb. 1994.

[2] J. Beran, R. Sherman, M. S. Taqqu, and W. Willinger, "Long-range dependence in variable-bit-rate video traffic," IEEE Trans. Communications, vol. 43, no. 2/3/4, pp. 1566-1579, Feb./Mar./Apr. 1995.

[3] O. Rose, "Statistical properties of MPEG video traffic and their impact on traffic modeling in ATM systems," University of Würzburg, Institute of Computing Science, Report No. 101, Feb. 1995.

[4] M. Reisslein et al., "Traffic and quality characterization of scalable encoded video: a large-scale trace-based study, part 2: Statistical analysis of single-layer encoded video," Arizona State University, Telecommunications Research Center, Tech. Rep., Jan. 2003.

[5] P. Abry, P. Flandrin, M. S. Taqqu, and D. Veitch, "Wavelets for the analysis, estimation, and synthesis of scaling data," in Self-similar Network Traffic and Performance Evaluation, edited by K. Park and W. Willinger. New York, NY: Wiley, 2000, pp. 39-88.

[6] P. Abry and D. Veitch, "Wavelet analysis of long-range dependent traffic," IEEE Trans. Information Theory, vol. 44, no. 1, pp. 2-15, Jan. 1998.

[7] F. Xue and Lj. Trajković, "Performance analysis of a wavelet-based Hurst parameter estimator for self-similar network traffic," in Proc. SPECTS, Vancouver, BC, May 2000, pp. 294-298.

[8] K. Park and W. Willinger, "Self-similar network traffic: an overview," in Self-similar Network Traffic and Performance Evaluation, edited by K. Park and W. Willinger. New York, NY: Wiley, 2000, pp. 1-38.

[9] University of Würzburg, Index of MPEG traces [Online]. Available: http://www-info3.informatik.uni-wuerzburg.de/MPEG/traces/.

[10] University of Berlin, MPEG-4 and H.263 video traces for network performance evaluation [Online]. Available: http://www-tkn.ee.tu-berlin.de/research/trace/trace.html.

[11] O. Rioul and M. Vetterli, "Wavelets and signal processing," IEEE Signal Processing Mag., vol. 8, no. 4, pp. 14-38, Oct. 1991.

[12] D. Veitch, MATLAB code for estimation of scaling exponents [Online]. Available:

http://www.cubinlab.ee.mu.oz.au/ darryl/secondorder_code.html.

[13] D. Veitch, MATLAB code for the estimation of multiscaling exponents [Online]. Available: http://www.cubinlab.ee.mu.oz.au/ darryl/MS_code.html.

[14] D. Veitch and P. Abry, "A statistical test for the time constancy of scaling exponents," IEEE Trans. Signal Processing, vol. 49, no. 10, pp. 23252334, Oct. 2001.

[15] M. S. Taqqu, Statistical methods for long-range dependence [Online]. Available: http://math.bu.edu/people/murad/methods/index.html.

[16] Engineering statistics [Online]. Available: http://www.itl.nist.gov/div898/handbook/. 